\title{
ON THE SOLITARY WAVE DYNAMICS OF TENSEGRITY LATTICES WITH STIFFENING RESPONSE: A NUMERICAL STUDY
}

\author{
Andrea Micheletti, Giuseppe Ruscica, ${ }^{2}$ Ada Amendola, ${ }^{3}$ Ida Mascolo ${ }^{3}$, FernandoFraternali ${ }^{3}$ \\ ${ }^{1}$ Department of Civil and Computer Science Engineering, University of Rome Tor Vergata, Italy \\ e-mail: micheletti@ing.uniroma2.it \\ ${ }^{2}$ Department of Engineering and Applied Sciences, University of Bergamo, Italy \\ e-mail: giuseppe.ruscica@unibg.it \\ ${ }^{3}$ Department of Civil Engineering, University of Salerno, Italy \\ e-mail: \{adamendola1,imascolo,f.fraternali $\} @$ unisa.it
}

Keywords: Tensegrity lattices, Stiffening behavior, Solitary waves, Compactons, Acoustic metamaterials.

\begin{abstract}
We present some peculiar results about the solitary-wave dynamics of novel tensegritybased metamaterials. It has been previously shown that one-dimensional chains of triangular tensegrity prisms with stiffening behavior support the propagation of compressive solitary waves. We show that such result can be generalized to two-dimensional and three-dimensional modular tensegrity lattices composed of polygonal and polyhedral units. Differently from the one-dimensional case, the stiffening response of these lattices originates at the interface between adjacent units, not from the unit themselves. We present numerical results on the response to impulsive loads of slender assemblies composed by square units in two-dimensions, and cubic units in three-dimensions. We observed compact compressive waves forming at impact locations, together with localized thermalization effects. Such compact waves propagate with nearly constant speed and energy, while maintaining their shape, and emerge from collision with other compact waves almost unaltered, losing a small fraction of their energy. These results suggest the investigation of the dynamics of regular and quasi-regular tessellations formed by other types of polygonal and polyhedral units.
\end{abstract}




\section{INTRODUCTION}

Nonlinear tensegrity metamaterials based on triangular tensegrity prisms have been proposed in the recent years as candidate for novel acoustic devices, owing to their ability to support the propagation of both compression and rarefaction solitary waves [12, 15, 16]. Such ability depends in turn on the possibility of designing tensegrity prisms with either stiffening or softening elastic response to external loads, by a suitable choice of geometry and prestress parameters [13, 14].

In this work we present some numerical investigations on the behavior of novel multidimensional tensegrity beams, more precisely, two-dimensional and three-dimensional slender modular assemblies with stiffening behavior composed by selected tensegrity units. We observed that compressive solitary waves appears also in these systems when subjected to impulsive loads.

In the next section, we describe the peculiar geometry and the origin of stiffening behavior of the structures we consider, together with our modeling assumptions. In Section 3 we present the results of numerical experiments. Our concluding remarks are given in Section 4.

\section{INTERFACE-LEVEL STIFFENING BEHAVIOR AND MODELING ASSUMPTIONS}

Differently from the one-dimensional case described in [12, 15, 16], the polygonal and polyhedral units we consider (Figs. 1, 2) do not feature a stiffening behavior by themselves; instead, such behavior results from the internal mechanism which is formed at the interface between adjacent units in modular assemblies, as explained below.

By considering the large-displacement static response of the two-cable systems in Fig. 3 (left) when subjected to a transverse load, it is easy to see that the load-vs-displacement relationship is nonlinear, and it is represented by a stiffening cubic curve with the inflection point located at the origin, Fig. 3 (right), with the slope at the inflection point being proportional to the prestress initially present in the system.

By connecting several units together as in Figs. 1 and 2, two-cable subsystems similar to the one in Fig. 3 are formed between adjacent units, conferring to the assembly the anticipated stiffening behavior when subjected to external actions.
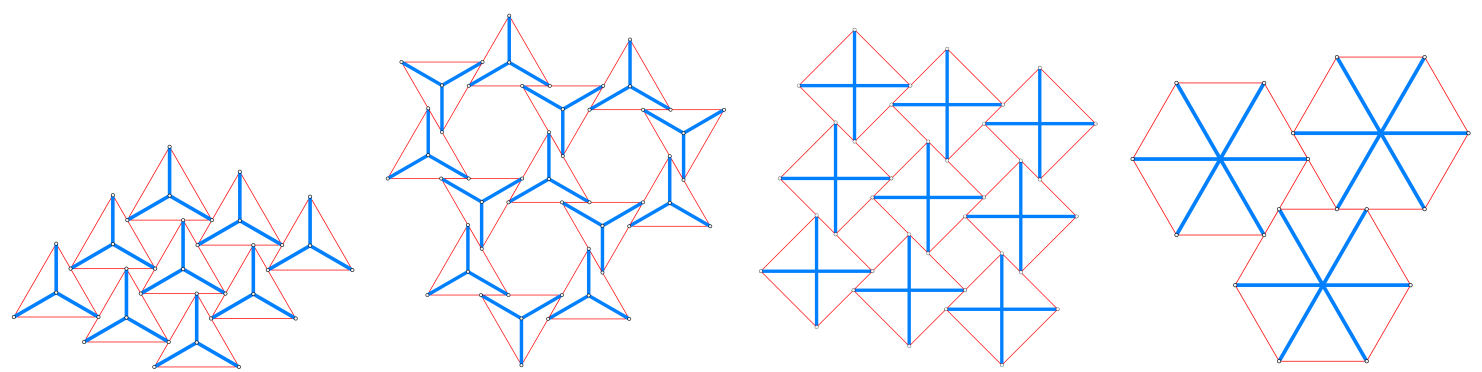

Figure 1: Two-dimensional assemblies: regular assemblies of polygonal cells.

We make the following assumptions: bars can resist both tension and compression while cables can resist tension only; bars and cables are linearly elastic; mass is lumped at bars' endpoints; there is no dissipation. We perform computations in a regime of large displacements by integrating numerically the (second-order nonlinear) equation of motion (cf. [11])

$$
\mathbf{M} \ddot{\mathbf{x}}+\mathbf{A}(\mathbf{x}) \mathbf{t}(\mathbf{x})=\mathbf{0}
$$



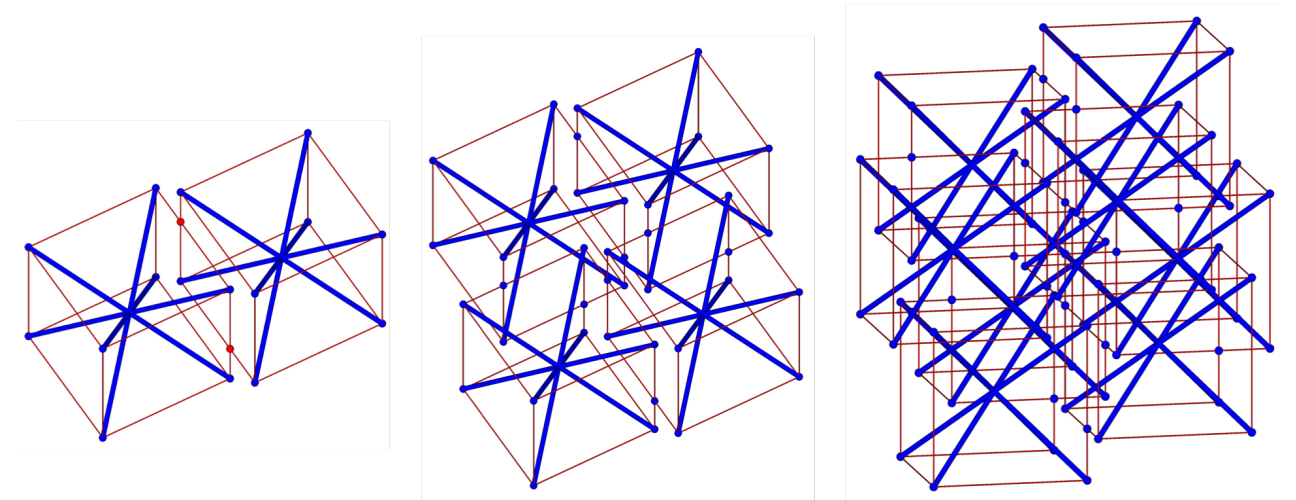

Figure 2: Three-dimensional assemblies: connecting two cubic cells at intermediate nodes (left); a $2 \times 2 \times 1$ (center) and a $2 \times 2 \times 2$ (right) assembly of cubic cells.
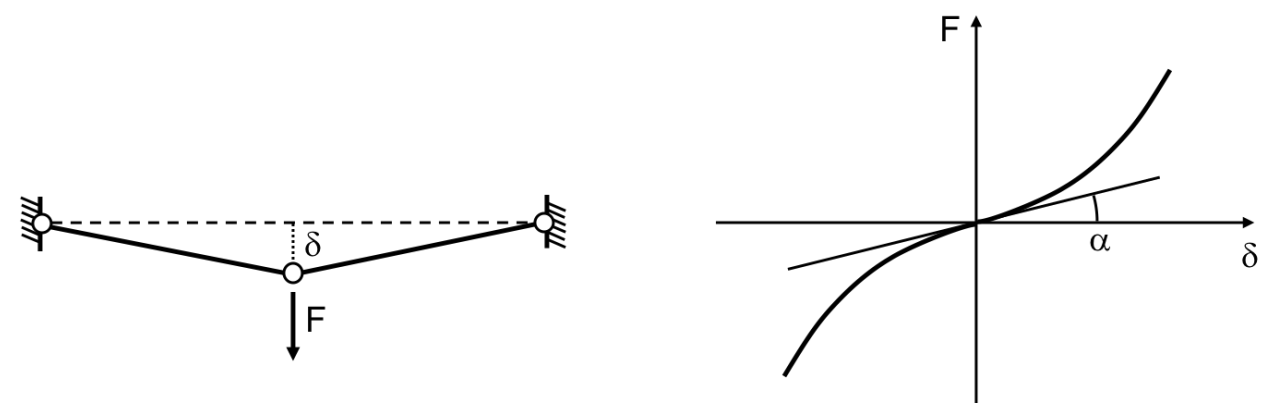

Figure 3: Nonlinear stiffening response of a two-string system: two-string systems subjected to a transverse load (left); force vs. displacement relationship (right). The slope $\tan \alpha$ of the tangent at the origin is directly proportional to the prestressing force.

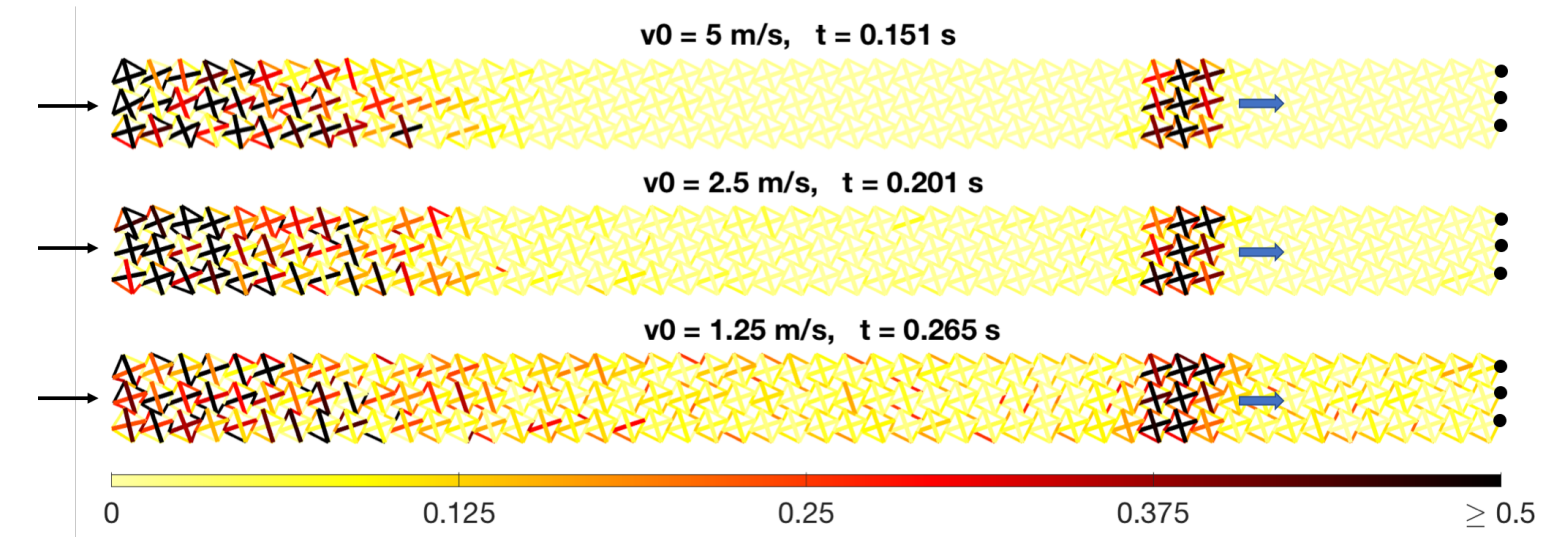

Figure 4: Deformed configuration and colormap of the elements' total energy after impact for different value of $v_{0}$. 
with assigned initial conditions. In (1), $\mathrm{x}$ is the vector of the $3 n$ nodal coordinates, with $n$ the number of nodes, $\mathbf{M}$ is the (constant) mass matrix, $\mathbf{A}$ is the equilibrium matrix, and $\mathbf{t}$ is the vector of the $e$ elements' axial forces, with $e$ the total number of elements.

The above equation of motion is solved in Matlab ${ }^{\circledR}$ by using the built-in routine ode45.

\section{NUMERICAL EXPERIMENTS ON 2D AND 3D TENSEGRITY BEAMS}

In this section's examples, we adopt the following geometric and material properties. The units' side length is $20 \mathrm{~mm}$; bars have a diameter of $1.75 \mathrm{~mm}$ and are realized in titanium alloy $\mathrm{Ti}_{6} \mathrm{Al}_{4} \mathrm{~V}$ with Young's modulus equal to $120 \mathrm{GPa}$ and mass density of $4.42 \mathrm{~g} / \mathrm{cm}^{3}$; cables are realized in Nylon 12 fibers and have a diameter of $0.25 \mathrm{~mm}$ with a Young's modulus of $500 \mathrm{MPa}$ and a yield strain of over $30 \%$ (with a mass density of $1.03 \mathrm{~g} / \mathrm{cm}^{3}$ ) [27]. Extra mass is added to the structure by attaching spheres of radius $2.5 \mathrm{~mm}$ in lead, with mass density equal to $11.34 \mathrm{~g} / \mathrm{cm}^{3}$, to the bars endpoints. Cables initially carry a nearly null axial force, with a prestrain assigned equal to $10^{-5}$.

We consider first a cantilevered rectangular $3 \times 50$ assembly of square units constrained at one end and subjected to an impact loading at the other end (indicated, respectively, by black dots and a thin arrow in Fig. 4). A horizontal initial velocity of magnitude $v_{0}$ is assigned to the four nodes of the middle module at the impacted side. Simulation results are show in Fig. 4 as energy colormaps at a certain time instant. Each bar is assigned its elastic energy plus the kinetic energy relative to the lumped mass at the bar's end nodes, while each cable is assigned just its elastic energy. Each energy value is then expressed as a percentage of the total energy of the system.

Simulation results shows two distinct effects (Fig. 4): $(i)$ a thermalization effect in the region near the impacted side, where a portion of the impact energy spreads around in a chaotic motion, and $(i i)$ the appearance of a solitary compression wave with compact support, or compacton, originating from the impacted side and traveling toward the constrained side. The compacton spans about three units in the longitudinal directions and maintains its shape and traveling speed nearly unaltered. Figure 4 shows a comparison of the effects of impacts of different magnitude, i.e, obtained by assigning different value to $v_{0}$. In the three cases shown, we see that the traveling time (traveling speed) decreases (increases) in a nonlinear way when $v_{0}$ increases.

Our second example regards the collision of two compactons on a $2 \times 80$ assembly of square units (Fig. 5). Initial velocities of magnitude $v_{0}$ and opposite directions are assigned to the middle module nodes at both ends of the assembly. Two compactons originate from the thermalization regions near impact locations. Figure 5(top) shows the energy colormaps, obtained as in the previous case, before and after collision. Figure 5 (bottom) shows three snapshots of the horizontal component of the velocity of the nodes which are located along the blue (grey) line on the top panel of Fig. 5. The compactons emerge from collision nearly unaltered in shape, while each compacton's energy slightly decreases, passing from from $30.28 \%$ to $28.27 \%$. The energy is lost in a slight thermalization of the center region of the assembly. As to the horizontal component of nodal velocity, we see from Fig. 5(bottom) that it remains almost the same after collision, while thermalization motion spreads away from the center.

In the third example we simulated a $2 \times 2 \times 20$ assembly of cubic units with a setup similar to that of the first example (Fig. 6). An initial velocity $v_{0}$ is imposed to the nodes of the leftmost modules, while the rightmost nodes are constrained not to move. Energy colormaps are computed in the same way as in the previous examples and are shown in Fig. 6 for four different time instant, before and after reflection at the constrained end. We observe the same effects as in the two-dimensional example: thermalization near the impacted side and formation of a compact 


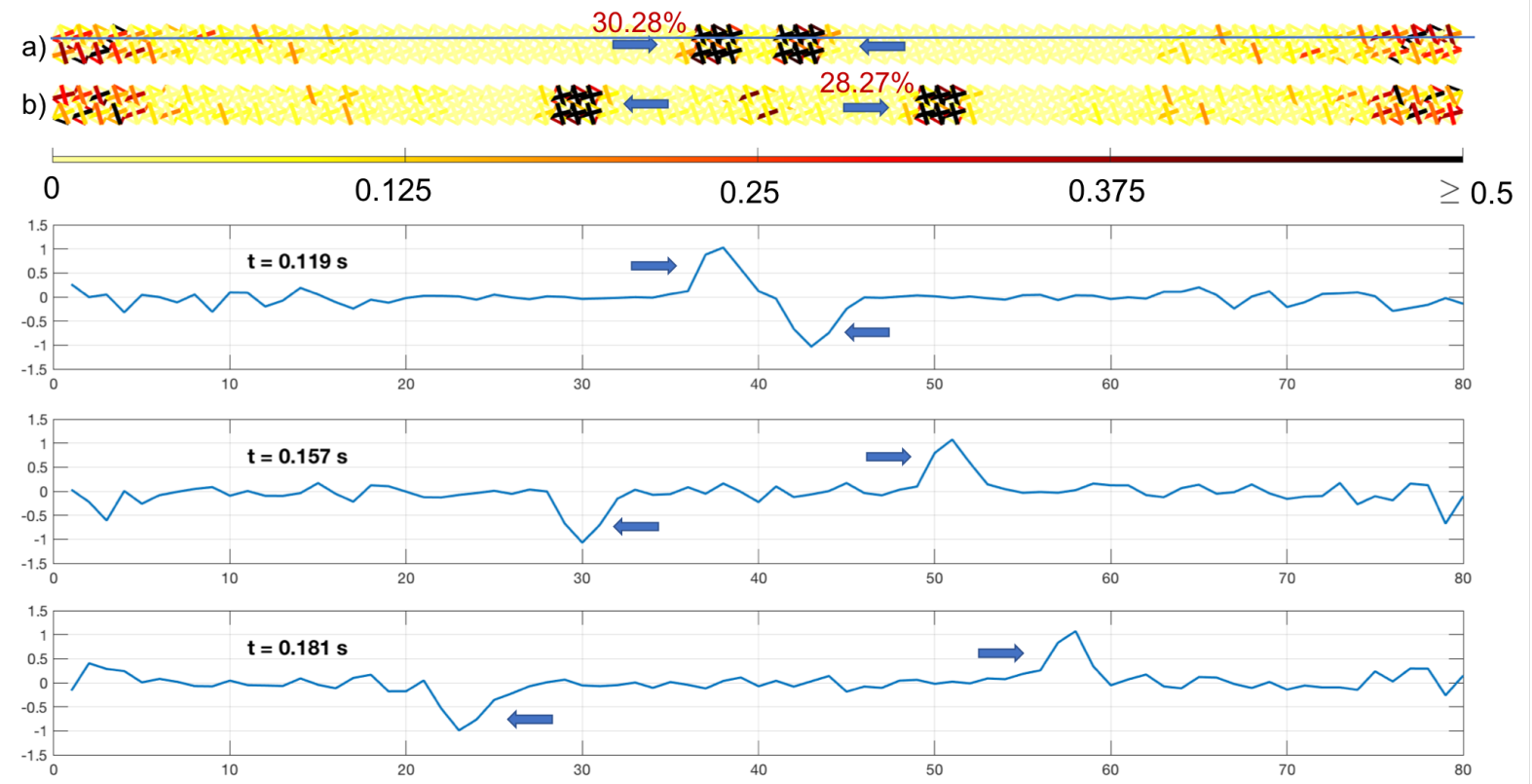

Figure 5: Deformed configuration and colormap of the elements' total energy.

compression solitary wave which maintains its shape and traveling speed nearly unaltered. In addition, after wave reflection, we observe some thermalization effects at the constrained end.

\section{CONCLUSIONS}

We studied the solitary-wave dynamics of tensegrity-based metamaterials. We showed that results previously obtained for one-dimensional chains of tensegrity prisms, regarding the formation of compression solitary waves by impact loading, can be generalized to two-dimensional and three-dimensional assemblies of polygonal and polyhedral units, where the stiffening behavior originated at the interface between adjacent units. We observed the formation of compact compression waves emerging from a thermalization region at the impact location. Such compact waves maintain their shape, energy, and traveling speed nearly unaltered during propagation, with slight losses after collision with another compact wave and after reflection. In a future study we envisage to combine together different polygonal (polyhedral) units so as to form regular or quasi-regular assemblies to obtain systems with wave-steering properties and to search for other peculiar behaviors.

\section{ACKNOWLEDGEMENTS}

AM and GR gratefully acknowledge the financial support from the Italian Ministry of Education, University, and Research (MIUR) under the 'FFABR' grant L.232/2016. AA, IM, and FF gratefully acknowledges financial support from the Italian Ministry of Education, University, and Research (MIUR) under the 'Departments of Excellence' grant L.232/2016.

\section{REFERENCES}

[1] M.H. Lu, L. Feng, Y.F. Chen, Phononic crystals and acoustic metamaterials. Mater. Today 12, 34-42, 2009. 

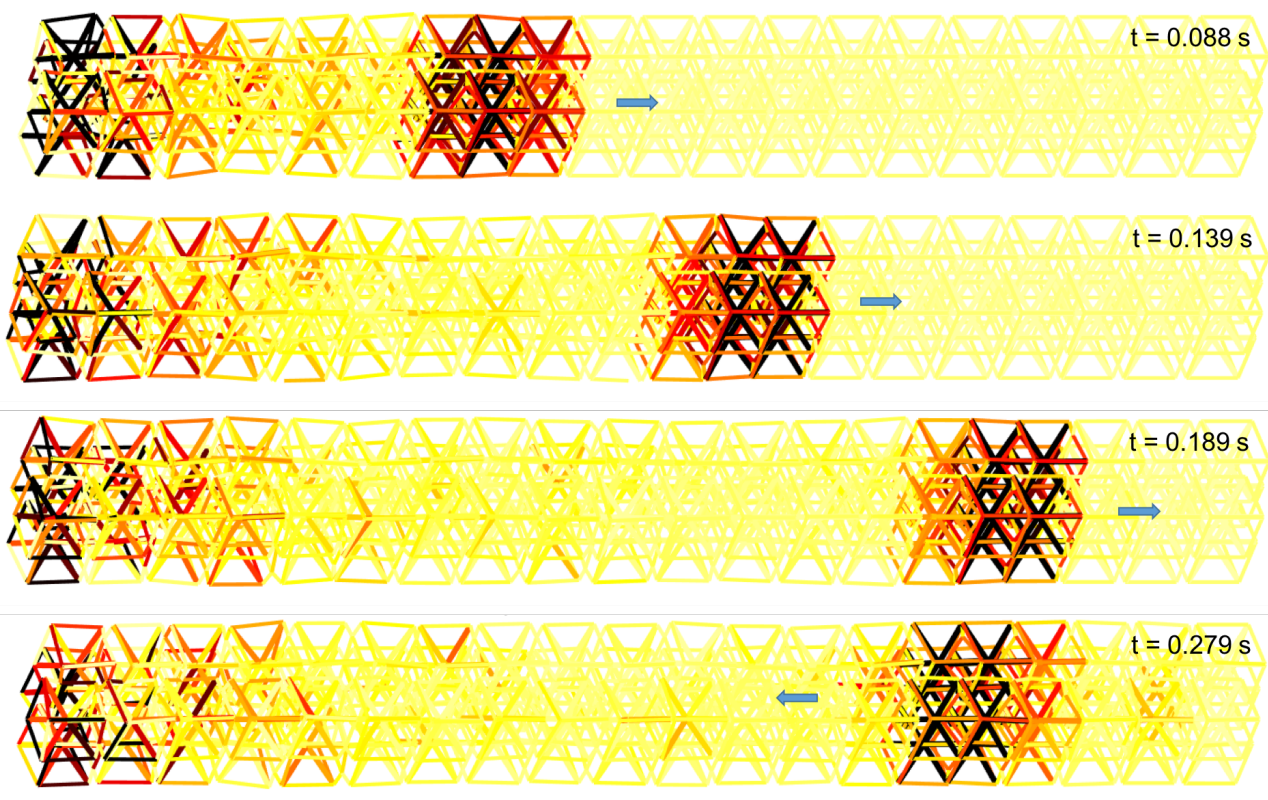

$\begin{array}{lllll}0 & 0.035 & 0.07 & 0.105 & \geq 0.14\end{array}$

Figure 6: Deformed configurations with superimposed colormaps of elements' energy fractions at different times after impact with initial velocity $v_{0}=1.25 \mathrm{~m} / \mathrm{s}$, on a $2 \times 2 \times 20$ beam of cubic cells.

[2] Maldovan, M. (2013), Sound and heat revolution in phononics. Nature, 503, 209-217.

[3] Phani, A. S., Hussein, M. I. (eds) (2017). Dynamics of Lattice Materials, John Wiley \& Sons, Ltd, Chichester, UK.

[4] Meza, L.R., Das, S., Greer, J.R. (2014). Strong, lightweight, and recoverable threedimensional ceramic nanolattices, Science 345 (6202), 1322-1326.

[5] Zheng, X. et al. (2014). Ultralight, ultrastiff mechanical metamaterials, Science, 344, 6190.

[6] Brunet, T., Leng, J., Mondain-Monva, O. (2013). Soft acoustic metamaterials. Science, 342, 323-324.

[7] Liu, Z., Zhang, X., Mao, Y., Zhu, Y. Y., Yang, Z., Chan, C. T., Sheng, P. (2000). Locally resonant sonic materials. Science 289, 1734-1736.

[8] Mitchell, S.J., Pandolfi, A. Ortiz, M, 2014. Metaconcrete: designed aggregates to enhance dynamic performance, J. Mech. Phys. Solids, 65, 69-81.

[9] Theocharis, G., Boechler, N., Daraio, C. (2013), Nonlinear phononic structures and metamaterials, in P.A. Deymier (ed.) Acoustic Matematerials and Phononic Crystals, Springer Series in Solid State Sciences, 173.

[10] Skelton, R.E., de Oliveira, M.C. (2010). Tensegrity Systems. Springer, Berlin.

[11] Sultan, C., Skelton, R. E. (2003). Deployment of tensegrity structures. Int. J. Solid Struct. 40, 4637-4657 
[12] Fraternali, F., Senatore, L., Daraio, C. (2012). Solitary waves on tensegrity lattices. J. Mech. Phys. Solids 60, 1137-1144.

[13] Fraternali, F., Carpentieri, G., Amendola, A. (2014). On the mechanical modeling of the extreme softening/stiffening response of axially loaded tensegrity prisms. J. Mech. Phys. Solids 74, 136-157.

[14] Amendola, A., Carpentieri, G., De Oliveira, M., Skelton, R.E., Fraternali, F. (2014). Experimental investigation of the softening-stiffening response of tensegrity prisms under compressive loading. Compos. Struct. 117, 234-243.

[15] Fraternali, F., Carpentieri, G., Amendola, A., Skelton, R.E., Nesterenko, V.F. (2014). Multiscale tunability of solitary wave dynamics in tensegrity metamaterials. Appl. Phys. Lett. 105, 201903.

[16] Davini, C., Micheletti, A., Podio-Guidugli, P. (2016). On the impulsive dynamics of T3 tensegrity chains. Meccanica 51 (11), 2763-2776.

[17] Amendola, A., Hernández-Nava, E., Goodall, R., Todd, I., Skelton, R.E., Fraternali, F. (2015). On the additive manufacturing, post-tensioning and testing of bi-material tensegrity structures. Compos. Struct. 131, 66-71.

[18] Tibert, A.G., Pellegrino, S., 2011. Review of form-finding methods for tensegrity structures. Int. J. Space Struct. 18, 209-223.

[19] Daraio, C.; Ngo, D, Nesterenko, V. F., Fraternali, F., 2010. Highly Nonlinear Pulse Splitting and Recombination in a Two Dimensional Granular Network. Phys Rev. E, 82, 036603.

[20] Fraternali, F., Porter, M.A., Daraio C. (2010). Optimal design of composite granular protector. Mechanics of Advanced Materials and Structures, 17, 1-19.

[21] Leonard, A. and Fraternali, F. and Daraio, C., 2013. Directional wave propagation in a highly nonlinear square packing of spheres. Exp. Mech. 53(3), 327-337.

[22] Ngo, D, Fraternali, F., Daraio, C., 2012. Highly Nonlinear Solitary Wave Propagation in Y-Shaped Granular Crystals with Variable Branch Angles. Phys Rev. E, 85, 036602-1-10.

[23] Nesterenko, V.F. (2001). Dynamics of Heterogeneous Materials, Springer-Verlag, New York.

[24] Herbold, E. B., Nesterenko, V. F. (2012). Propagation of rarefaction pulses in discrete materials with strain-softening behavior. Phys. Rev. Lett. 110, 144101.

[25] Amendola, A., Krushynska A., Daraio C., Pugno N.M., Fraternali F. (2018). Tuning frequency band gaps of tensegrity metamaterials with local and global prestress, Int. J. Solids Struct., 155, 47-56.

[26] Daraio, C., Fraternali, F. (2013). Method and Apparatus for Wave Generation and Detection Using Tensegrity Structures, US Pat. No. 8,616,328, granted on December 31, 2013 
[27] Materials Spotlight: The Properties of Nylon 12. Available at: https://www.cableorganizer.com/learning-center/articles/materials-nylon12.html. Date accessed: 14 Jan. 2019. 